\title{
Probing the internal magnetism of stars using asymptotic magneto-asteroseismology
}

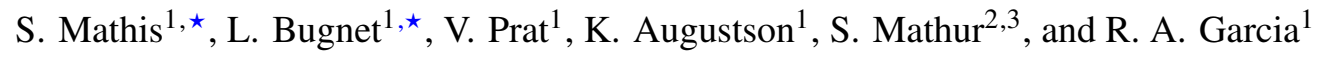 \\ 1 AIM, CEA, CNRS, Université Paris-Saclay, Université Paris Diderot, Sorbonne Paris Cité, 91191 Gif-sur-Yvette Cedex, France \\ e-mail: stephane.mathis@cea.fr,lisa.bugnet@cea.fr \\ 2 Instituto de Astrofísica de Canarias, 38200 La Laguna, Tenerife, Spain \\ 3 Universidad de La Laguna, Dpto. de Astrofísica, 38205 La Laguna, Tenerife, Spain
}

Received 13 August 2020 / Accepted 23 November 2020

\begin{abstract}
Context. Our knowledge of the dynamics of stars has undergone a revolution through the simultaneous large amount of high-quality photometric observations collected by space-based asteroseismology and ground-based high-precision spectropolarimetry. They allowed us to probe the internal rotation of stars and their surface magnetism in the whole Hertzsprung-Russell diagram. However, new methods should still be developed to probe the deep magnetic fields in these stars.

Aims. Our goal is to provide seismic diagnoses that allow us to probe the internal magnetism of stars.

Methods. We focused on asymptotic low-frequency gravity modes and high-frequency acoustic modes. Using a first-order perturbative theory, we derived magnetic splittings of their frequencies as explicit functions of stellar parameters.

Results. As in the case of rotation, we show that asymptotic gravity and acoustic modes can allow us to probe the different components of the magnetic field in the cavities in which they propagate. This again demonstrates the high potential of using mixed-modes when this is possible.
\end{abstract}

Key words. asteroseismology - waves - stars: magnetic field - stars: oscillations - methods: analytical

\section{Introduction}

Along with rotation, magnetism is one of the two corner stones of stellar dynamics. Magnetism deeply modifies the interactions of every type of stars with their surrounding environment and their evolution, in particular through the transport of angular momentum and chemicals that they potentially trigger in their interiors (e.g. Brun \& Browning 2017; Braithwaite \& Spruit 2017). In this framework, two revolutions have occurred in stellar physics in the past two decades.

The first revolution is space-based helio- and asteroseismology (e.g. Christensen-Dalsgaard 2015; García \& Ballot 2019; Aerts 2021). These techniques have allowed us to probe the structure, the internal rotation, and the magnetic activity of the Sun and of stars with high precision. Helioseismology analyses with spaced-based instruments (i.e. the Michelson Doppler Imager (MDI; Scherrer et al. 1995) and Global Oscillations at Low Frequencies (GOLF; Gabriel et al. 1995) instruments onboard the SOlar and Heliospheric Observatory (SOHO; Domingo et al. 1995) spacecraft) revealed the solar rotation profile down to $0.25 R_{\odot}\left(R_{\odot}\right.$ is the radius of the Sun) with an outer radiative core that rotates as a solid body. Strong efforts are still ongoing to constrain the rotation of the nuclear core (e.g. García et al. 2007; Mathur et al. 2008). Space-based asteroseismology with the Kepler space mission then allowed us to probe the rotation of stars from their surface to their core in the case of main-sequence and evolved low- and intermediatemass stars (Aerts et al. 2019, and references therein). First, acoustic modes have been used to study the internal rotation

\footnotetext{
^ S. Mathis and L. Bugnet equally contributed to this work.
}

of main-sequence low-mass stars (e.g. Benomar et al. 2015). Next, gravito-inertial modes allowed us to determine the rotation near the interface of the radiative envelope with the convective core and in the convective core of fast-rotating intermediatemass stars (e.g. Van Reeth et al. 2016; Ouazzani et al. 2020, respectively). Finally, mixed gravito-acoustic modes in evolved low- and intermediate-mass stars provide precise constraints on the rotation of the internal radiative zone down to the core and the averaged rotation of the envelope (e.g. Mosser et al. 2012; Gehan et al. 2018; Deheuvels et al. 2014, 2020). These observations have revealed core-to-surface rotation ratios that are weaker by several orders of magnitude than those predicted by the most modern rotating stellar models for the whole Hertzsprung-Russel diagram (e.g. Eggenberger et al. 2012; Ceillier et al. 2013; Marques et al. 2013; Cantiello et al. 2014; Ouazzani et al. 2019). This demonstrates that a powerful mechanism acts throughout the evolution of stars, including our Sun, and extracts angular momentum from the deep interior. The transport induced by stable (e.g. Mestel \& Weiss 1987; Mathis \& Zahn 2005) or unstable magnetic fields in stably stratified stellar radiation zones is one of the potential mechanisms (e.g. Spruit 2002; Fuller et al. 2019). These discoveries have triggered the development of a strong theoretical basis from which we interpret seismic observations. For oscillation modes, with frequencies far higher than the rotation frequency, the perturbative theory allows us to compute frequency splittings due to the Coriolis and centrifugal accelerations (e.g. Aerts et al. 2010; Goupil et al. 2013, and references therein). It also allows us to compute splittings due to the change of reference frame from the stellar corotating one to the observer frame. These splittings are then used to constrain the internal stellar rotation. This method 
has been intensively used to probe the solar rotation profile (e.g. Thompson et al. 1996; Couvidat et al. 2003) and the coreto-surface rotation ratio in evolved stars (e.g. Beck et al. 2012; Deheuvels et al. 2012, 2014, 2015, 2020; Spada et al. 2016). For low-frequency gravito-inertial modes with frequencies of the same order of magnitude as the rotation frequency, the traditional approximation of rotation (TAR), which is not perturbative, can be used (e.g. Lee \& Saio 1997; Townsend 2003; Van Reeth et al. 2018; Mathis \& Prat 2019). The traditional approximation of rotation can be used when the buoyancy-restoring force dominates the Coriolis force in the direction of the stable entropy and chemical stratification (Mathis 2009). When the variation in the period spacing (i.e. the difference between the periods of two consecutive modes) is considered as a function of the mode period, the (differential) rotation of the oscillations propagation cavity can be constrained (Bouabid et al. 2013; Van Reeth et al. 2018). This method is currently used to provide key information on the rotation rate of the radiative layers surrounding the convective core in fast-rotating intermediatemass stars (e.g. Van Reeth et al. 2016; Christophe et al. 2018; Aerts et al. 2019; Li et al. 2019, 2020). For each case, the study of the effect of rotation on asymptotic gravity (and gravitoinertial), acoustic, and mixed gravito-acoustic modes, which rapidly oscillate in the radial direction, has provided a deep understanding of their modification by the Coriolis (and centrifugal) acceleration and key tools for seismic modelling and inversions.

The second revolution is ground-based high-precision spectropolarimetry that probes the strength and geometric configuration of magnetic fields at the surface of stars in the whole Hertzsprung-Russell diagram from the pre-main sequence to the late stages of their evolution (e.g. Donati \& Landstreet 2009; Aurière et al. 2015; Wade et al. 2016). Large spectropolarimetric surveys thus led our knowledge of dynamo-generated and fossil magnetic fields to a new level of understanding, in particular through the simultaneous development of theoretical models and three-dimensional (3D), global, nonlinear magnetohydrodynamical (MHD) simulations (e.g. Brun \& Browning 2017; Braithwaite \& Spruit 2017). However, spectropolarimetry does not give access to the distribution of the magnetic field inside stars. Once again, asteroseismology is the answer. The method is the same as for rotation, that is, specific signatures of the effect of magnetism on the excitation, the propagation, and the damping of stellar oscillations are looked for. First studies have considered the effects of simple magnetic configurations such as purely dipolar poloidal fields (aligned or inclined to the rotation axis) or purely toroidal fields (e.g. Goode \& Thompson 1992; Takata \& Shibahashi 1994). However, our knowledge of magnetic topologies and their stability has strongly progressed in the past two decades through the simultaneous development of stellar spectropolarimetry and of 3D MHD simulations. For instance, we now have a better understanding of the formation and the topologies of fossil fields in stellar radiation zones (Braithwaite \& Spruit 2004). They should be a combination of dipolar poloidal and toroidal fields to be in their lowest energy stable equilibrium state (Duez \& Mathis 2010). Therefore it becomes mandatory to study the seismic signatures of such complex topologies. This has recently been undertaken for the fossil fields in the radiative envelope of upper main-sequence stars (Prat et al. 2019) and the radiative core of low-mass and intermediate-mass evolved stars (Bugnet et al. 2021) as well as for the dynamo fields generated in the convective envelope of solar-type stars (Kiefer \& Roth 2018).
In this framework, asymptotic analyses have been very useful to provide powerful seismic diagnoses for probing the rotation of stellar interiors (Goupil et al. 2013). In this article, we show how a method like this, which has been developed for rotation, can be applied to magnetism. In Sect. 2 we first recall the asymptotic theory for acoustic, gravity, and mixed gravitoacoustic modes. In Sect. 3 we identify the dominant terms in the magnetic splittings for each class of modes, and we derive asymptotic expressions that are the counterpart of those known for stellar rotation. In Sect. 4 we present a proof-of-concept application to a typical intermediate-mass red giant star. Finally, in Sect. 5, we make a synthesis of the results and discuss how asymptotic predictions can allow us to probe the distribution of the magnetic field at different depths in stars, and the applications of these predictions to different stellar types that can be foreseen.

\section{Asymptotic theory for (mixed) stellar oscillation modes}

We first recall the basics of the theory of the oscillations of nonrotating and non-magnetic stars, which is necessary to compute first-order magnetic splittings in the asymptotic limits of lowfrequency gravity $(g)$ modes and high-frequency pressure $(p)$ modes. The Lagrangian displacement of an oscillation eigenmode is expanded as

$\boldsymbol{\xi}(\boldsymbol{r}, t)=\operatorname{Re}\left\{\left[\xi_{\mathrm{r}}(r) Y_{l}^{m}(\theta, \varphi) \boldsymbol{e}_{\mathrm{r}}+\xi_{\mathrm{h}}(r) \boldsymbol{\nabla}_{\mathrm{h}} Y_{l}^{m}(\theta, \varphi)\right] e^{-i \omega t}\right\}$,

where $\xi_{\mathrm{r}}$ and $\xi_{\mathrm{h}}$ are the radial functions of its vertical and horizontal components, $\omega$ is the angular frequency, $Y_{l}^{m}$ are the spherical harmonics, $l$ is its degree, and $m$ the azimuthal order, $\boldsymbol{\nabla}_{\mathrm{h}}=\partial_{\theta}(\cdot) \boldsymbol{e}_{\theta}+1 / \sin \theta \partial_{\varphi}(\cdot) \boldsymbol{e}_{\varphi}$ is the horizontal gradient, $\left\{\boldsymbol{e}_{\mathrm{r}}, \boldsymbol{e}_{\theta}, \boldsymbol{e}_{\varphi}\right\}$ are the spherical unit vectors, and $\operatorname{Re}$ is the real part of a complex number. Following Gough (1993) and Hekker \& Christensen-Dalsgaard (2017), who used the adiabatic linearised hydrodynamical equations assuming the Cowling approximation, we derive the Schrödinger-like wave equation

$\frac{\mathrm{d}^{2} X}{\mathrm{~d} r^{2}}+k_{\mathrm{r}}^{2}(r) X=0$

where $X=c^{2} \rho^{1 / 2} \operatorname{div} \boldsymbol{\xi}$. The sound speed $c$ is defined as $c=$ $\sqrt{\Gamma_{1} P / \rho}$, where $\rho$ and $P$ are the density and the pressure of the hydrostatic background, respectively, and $\Gamma_{1}=(\partial \ln P / \partial \ln \rho)_{S}$ is the first adiabatic exponent, with $S$ being the macroscopic entropy. The vertical wave number $k_{\mathrm{r}}$ is given by

$k_{\mathrm{r}}^{2}=\frac{1}{c^{2}(r)}\left[S_{l}^{2}(r)\left(\frac{N^{2}(r)}{\omega^{2}}-1\right)+\omega^{2}-\omega_{\mathrm{c}}^{2}(r)\right]$.

We have identified the Lamb frequency $S_{l}$ defined by

$S_{l}^{2}=\frac{l(l+1) c^{2}}{r^{2}}=k_{\mathrm{h}}^{2} c^{2}, \quad$ where $\quad k_{\mathrm{h}}=\frac{\sqrt{l(l+1)}}{r}$

is the horizontal wave number, and the Brunt-Väisälä frequency $N$ defined by

$N^{2}=g\left(\frac{1}{\Gamma_{1}} \frac{\mathrm{d} \ln P}{\mathrm{dr}}-\frac{\mathrm{d} \ln \rho}{\mathrm{d} r}\right)$,

where $g$ is the gravity of the hydrostatic background. Finally, $\omega_{c}$ is the acoustic cut-off frequency, defined by

$\omega_{\mathrm{c}}^{2}=\frac{c^{2}}{4 H^{2}}\left(1-2 \frac{\mathrm{d} H}{\mathrm{~d} r}\right)$

where $H=-(\mathrm{d} \ln \rho / \mathrm{d} r)^{-1}$ is the density scale height. 


\subsection{Asymptotic g modes}

In the low-frequency regime for which $\omega \ll\left\{S_{l}, N\right\}$, the Lagrangian displacement becomes mostly horizontal (i.e. $\left.\left|\xi_{\mathrm{r}}\right| \ll\left|\xi_{\mathrm{h}}\right|\right)$ and the vertical wave number reduces to (e.g. Hekker \& Christensen-Dalsgaard 2017)

$k_{\mathrm{r}} \approx \frac{N}{\omega} \frac{\sqrt{l(l+1)}}{r}$.

Using the reduced canonical variable

$W=\rho^{1 / 2} \omega r^{2}\left(\frac{N^{2}}{\omega^{2}}-1\right)^{-1 / 2} \xi_{\mathrm{h}}$

which has been introduced by Shibahashi (1979), and applying the JWKB (for Jeffreys, Wentzel, Kramers, and Brillouin) method (we refer to Appendix $C$ and to Erdelyi 1956; Fröman \& Fröman 2005), we derive the asymptotic expression for $\xi_{\mathrm{h}}(r)$

$$
\begin{aligned}
\xi_{\mathrm{h}}= & A_{W} \rho^{-1 / 2} \omega^{-3 / 2} r^{-3 / 2}[l(l+1)]^{-1 / 4} N^{1 / 2} \\
& \times \sin \left(\int_{r_{t ; i}}^{r} k_{\mathrm{r}} \mathrm{d} r^{\prime}-\phi_{g}^{\prime}\right),
\end{aligned}
$$

where $A_{W}$ is the amplitude fixed by the excitation and damping mechanisms and the inertia of the mode (Samadi et al. 2015), $\phi_{g}^{\prime}$ is a phase, and $r_{t ; i}\left(r_{t ; e}\right)$ is the internal (external) turning point for which $k_{\mathrm{r}}$ vanishes.

\subsection{Asymptotic $p$ modes}

In the high-frequency regime for which $\omega \gg S_{l}$, the Lagrangian displacement becomes mostly vertical (i.e. $\left|\xi_{\mathrm{h}}\right|<\left|\xi_{\mathrm{r}}\right|$ ) and the vertical wave number reduces to

$k_{\mathrm{r}} \approx \frac{\omega}{c}$.

Using the reduced canonical variable

$V=\rho^{1 / 2} \operatorname{cr}\left(1-\frac{S_{l}^{2}}{\omega^{2}}\right)^{-1 / 2} \xi_{\mathrm{r}}$,

which has been introduced by Shibahashi (1979), and applying again the JWKB method (Appendix C), we derive the asymptotic expression for $\xi_{\mathrm{r}}(r)$

$\xi_{\mathrm{r}}=A_{V} \rho^{-1 / 2} c^{-1 / 2} \omega^{-1 / 2} r^{-1} \cos \left(\int_{\mathrm{r}}^{R} k_{\mathrm{r}} \mathrm{d} r^{\prime}-\phi_{\mathrm{p}}^{\prime}\right)$,

where $A_{V}$ is the mode amplitude, $\phi_{\mathrm{p}}^{\prime}$ is a phase, and $R$ is the radius of the star.

\section{Asymptotic magnetic splittings}

\subsection{Studied magnetic configuration}

For a sufficiently moderate magnetic field, we can perturb the wave equation to the first-order by the linearised Lorentz force

$\delta \boldsymbol{F}_{\mathrm{L}}=\frac{1}{4 \pi}[(\boldsymbol{\nabla} \wedge \boldsymbol{B}) \wedge \delta \boldsymbol{B}+(\boldsymbol{\nabla} \wedge \delta \boldsymbol{B}) \wedge \boldsymbol{B}]$,

where $\boldsymbol{B}$ is the stellar magnetic field, $\delta \boldsymbol{B}=\boldsymbol{\nabla} \wedge(\boldsymbol{\xi} \wedge \boldsymbol{B})$ is its fluctuation, and $\mu_{0}=4 \pi$ (in c.g.s.) is the magnetic permeability of vacuum. We obtain the general expression for the magnetic frequency splitting

$\left(\frac{\delta \omega_{\mathrm{mag}}}{\omega_{0}}\right)=-\frac{\left\langle\boldsymbol{\xi}, \delta \boldsymbol{F}_{\mathrm{L}} / \rho\right\rangle}{2 \omega_{0}\langle\boldsymbol{\xi}, \boldsymbol{\xi}\rangle}$,

where $\langle\boldsymbol{\xi}, \boldsymbol{\zeta}\rangle=\int_{V} \rho\left(\boldsymbol{\xi}^{*} \cdot \boldsymbol{\zeta}\right) \mathrm{d} V$ (where the asterisk is the complex conjugate) and $\omega_{0}$ is the angular frequency of the unperturbed mode; we refer to Prat et al. (2019) for details on the perturbation formalism. A supplementary term, $\boldsymbol{\nabla} \cdot(\rho \boldsymbol{\xi})(\boldsymbol{\nabla} \wedge \boldsymbol{B}) \wedge$ $\boldsymbol{B} /\left(\mu_{0} \rho\right)$, should be taken into account in the general case (Goode \& Thompson 1992). However, we can demonstrate again using the JWKB approximation that it is not the dominant term for the asymptotic modes. It scales as $k_{\mathrm{r}}$, while the dominant terms scales as $k_{\mathrm{r}}^{2}$ while $k_{r} H \gg 1$ in the JWKB limit. Therefore, we assume that the characteristic length scale of the wave is shorter than the length scales of the variation in the hydrostatic background $(H)$ and in the magnetic field configuration.

The mode inertia is expressed as

$\langle\boldsymbol{\xi}, \boldsymbol{\xi}\rangle=\int_{0}^{R} \rho r^{2}\left(\left|\xi_{\mathrm{r}}\right|^{2}+l(l+1)\left|\xi_{\mathrm{h}}\right|^{2}\right) \mathrm{d} r$.

In this work, we chose to focus on a dipolar mixed (i.e. with both a poloidal and a toroidal components) axisymmetric configuration

$\boldsymbol{B}=B_{0}\left[b_{\mathrm{r}}(r) \cos \theta, b_{\theta}(r) \sin \theta, b_{\varphi}(r) \sin \theta\right]$,

where $B_{0}$ is the field amplitude and the functions $b_{i}(r)$ (with $i=\{r, \theta, \varphi\})$ describe the radial dependence of each component. On the one hand, it can model a stable fossil field that has been formed in a stably stratified radiation zone (e.g. Duez \& Mathis 2010). On the other hand, it can model the first large-scale component of a dynamo-generated magnetic field (e.g. Brun \& Browning 2017).

\subsection{Asymptotic g modes}

We first focus on low-frequency asymptotic $g$ modes, which probe radiative zones, that is, cores of low-mass stars. For these modes, we have $\omega_{0} \ll\left\{S_{l}, N\right\}$ and thus $k_{\mathrm{r}} \approx\left(N / \omega_{0}\right) k_{\mathrm{h}} \gg k_{\mathrm{h}}$ with $k_{\mathrm{h}} \equiv \sqrt{l(l+1)} / r$. Because of their weak compressibility, $\boldsymbol{k} \cdot \boldsymbol{\xi} \approx$ 0 , and they are thus quasi-horizontal with $\xi_{\mathrm{v}} \ll \xi_{\mathrm{h}}$. Therefore we selected the terms that involve the products $\left\{\xi_{\mathrm{h}}, \xi_{\mathrm{h}}^{\prime}, \xi_{\mathrm{h}}^{\prime \prime}\right\} \times \xi_{\mathrm{h}}^{*}$ to isolate the dominant terms in the general expression of the magnetic splitting provided by Eqs. (B.1) and (B.2). Then, using the results provided in Eq. (9), we have $\xi_{\mathrm{h}} \propto\left(k_{\mathrm{r}}\right)^{-1 / 2} \exp \left[i \int k_{\mathrm{r}} \mathrm{d} r^{\prime}\right]$ if the studied low-frequency mode varies rapidly in space in comparison to the characteristic length scale of the variation in the stellar hydrostatic structure and in the magnetic configuration. We thus have $\xi_{\mathrm{h}}^{\prime} \propto i k_{\mathrm{r}} \xi_{\mathrm{h}}$ and $\xi_{\mathrm{h}}^{\prime \prime} \propto k_{\mathrm{r}}^{2} \xi_{\mathrm{h}}$. Because $k_{\mathrm{r}} H \gg 1$ (because $N \gg \omega$, and where $H$ is the characteristic length of the variation in the hydrostatic background) in the asymptotic regime, the dominant terms are thus those $\propto k_{\mathrm{r}}^{2}$, that is, $\propto \xi_{\mathrm{h}}^{\prime \prime} \xi_{\mathrm{h}}^{*}$. As in Hasan et al. (2005) and Rashba et al. (2007), we can therefore identify the dominant terms in the complete expression of the magnetic splitting given in Eq. (B.1). After a last integration by parts, we obtain

$\left(\frac{\delta \omega_{\mathrm{mag}}}{\omega_{0}}\right)_{g}=\frac{B_{0}^{2}}{8 \pi \omega_{0}^{2}} C_{l, m} \frac{\int_{r_{t ; i}}^{r_{t ; e}}\left|\left(r b_{\mathrm{r}} \xi_{\mathrm{h}}\right)^{\prime}\right|^{2} \mathrm{~d} r}{\int_{r_{t ; i}}^{r_{t ; e}}\left|\xi_{\mathrm{h}}\right|^{2} \rho r^{2} \mathrm{~d} r}$,

where 

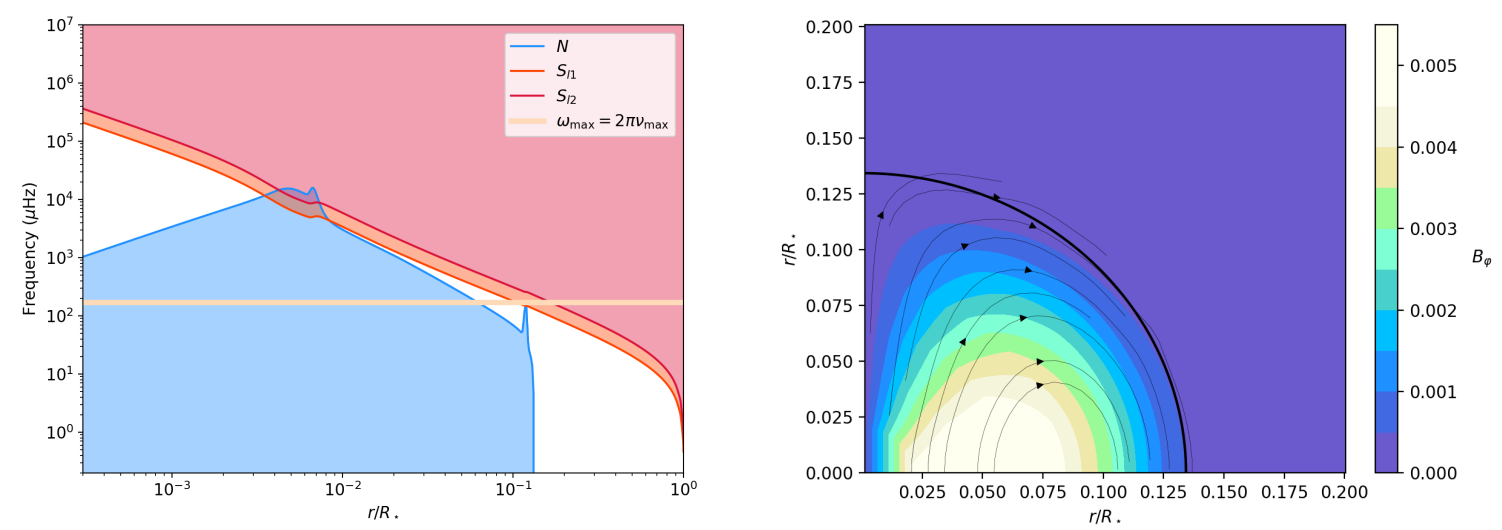

Fig. 1. Left: profiles of the Lamb frequencies $\left(S_{l}\right.$; red lines) for $l=\{1,2\}$ and of the Brunt-Väisälä frequency $\left(N\right.$; blue line) for the studied $1.5 M_{\odot}$ $2.8 \mathrm{Gyr}$ old intermediate-mass red giant star (with a solar metallicity $Z=0.02$ ); the position of $v_{\max }$ is also reported (orange line). Right: topology of the fossil magnetic field in the stably stratified radiative core of the studied red giant (its limit is given by the thick black line). The thin black lines are the poloidal field lines, while the intensity of the toroidal field (normalised by the maximum values of the radial field) is given in colours.

$$
C_{l, m}=\frac{\int_{0}^{\pi}\left[\left|\cos \theta \partial_{\theta} Y_{l}^{m}\right|^{2}+m^{2}\left|\frac{\cos \theta}{\sin \theta} Y_{l}^{m}\right|^{2}\right] \sin \theta \mathrm{d} \theta}{l(l+1)} .
$$

Because the angular coupling coefficient $C_{l, m}$ is independent of the sign of $m$ in the case of the considered dipolar magnetic configuration, we can already predict that the magnetic frequency splittings for pro- and retrograde modes $(m>0$ and $m<0$, respectively) will be the same. This will allow us to potentially distinguish them from the rotation-induced frequency splittings which depend on the sign of $m$ with opposite values when computed to the first-order in $\Omega$ ( $\Omega$ is the rotation of the star); this is discussed in details in Sect. 4. In the case of main-sequence lowmass stars and of evolved stars, we can assume that $r_{t ; i} \approx 0$ and $r_{t ; e} \approx R_{\text {core }}$ for low-frequency $g$ modes, where $R_{\text {core }}$ is the radius of the radiative core. In the case of main-sequence intermediatemass stars, we have $r_{t ; i} \approx R_{\mathrm{CC}}$ and $r_{t ; e} \approx R$, where $R_{\mathrm{CC}}$ is the radius of the convective core.

In addition, the mostly horizontal low-frequency $g$ modes allows us to probe the orthogonal radial component of the field. This can be understood from the form of the linearised Lorentz force, which implies terms of the form $\boldsymbol{\xi} \wedge \boldsymbol{B}$ (cf. Eq. (13)). These terms thus couple the Lagrangian displacement of the mostly horizontal mode with the orthogonal radial component of the field.

Using Eq. (9), this can be written as

$\left(\frac{\delta \omega_{\mathrm{mag}}}{\omega_{0}}\right)_{g}=\frac{1}{2 \omega_{0}^{4}} C_{l, m} \frac{\int_{r_{t ; i}}^{r_{t, e}}\left(\omega_{A}^{r}\right)^{2} N^{2} \cos ^{2}\left(X_{g}\right) N \frac{\mathrm{d} r}{r}}{\int_{r_{t, i}}^{r_{t, e}} N \frac{\mathrm{d} r}{r}}$,

where $X_{g}=\int_{r_{t i}}^{r} k_{\mathrm{r}} \mathrm{d} r^{\prime}-\phi_{g}^{\prime}$ and $\omega_{A}^{r}=B_{0} b_{\mathrm{r}} /(\sqrt{4 \pi \rho} r)$.

Using the properties of rapidly oscillating integrals (see Appendix C), this reduces to

$\left(\frac{\delta \omega_{\mathrm{mag}}}{\omega_{0}}\right)_{g}=\frac{1}{2} \frac{B_{0}^{2}}{4 \pi \rho_{c} R^{2} \omega_{0}^{2}} \frac{N_{\max }^{2}}{\omega_{0}^{2}} l(l+1) C_{l, m} \frac{\int_{x_{t ; i}}^{x_{t ; e}} \frac{b_{\mathrm{r}}^{2} \widehat{N}^{2}}{\left(\rho / \rho_{c}\right) x^{2}} \widehat{N} \frac{\mathrm{d} x}{x}}{\int_{x_{t ; i}}^{x_{t ; e}} \widehat{N} \frac{\mathrm{d} x}{x}}$,

where we have introduced the dimensionless radius $x=r / R$ (with $x_{t ; i}=r_{t ; i} / R$ and $x_{t ; e}=r_{t ; e} / R$ ), the maximum of the BruntVäisälä frequency $N_{\max }$ and its dimensionless radial profile $\widehat{N}$ defined such that $N(r)=N_{\max } \widehat{N}(r)$. The form of this integral is really interesting because we recover the one known for asymptotic rotational splittings, but for the radial component of the magnetic field. We can then define a kernel as Goupil et al. (2013) (see the end of Sect. 3.4.), which opens the path for potential inversions when magnetic seismic signatures can be detected (we refer to Bugnet et al. 2021 for the corresponding values of the critical amplitudes).

This result can be generalised for $\mathrm{g}$ modes with frequencies of the same order of magnitude as the inertial frequency $2 \Omega$, where $\Omega$ is the angular velocity of the star. This is the case for instance in fast-rotating stars such as $\gamma$ Doradus stars (e.g. Van Beeck et al. 2020) and SPB stars (e.g. Prat et al. 2019). When the buoyancy force exceeds the Coriolis force in the direction of the stable entropy or chemical stratification, we can assume the traditional approximation of rotation (TAR; e.g. Eckart 1960; Lee \& Saio 1997; Townsend 2003; Bouabid et al. 2013; Mathis 2009). In this approximation, the horizontal projection of the rotation vector is neglected that allows us to separate variables when the wave propagation equation is solved as in the non-rotating case. Then, we obtain for $g$ modes modified by rotation, in other words, for gravito-inertial modes (hereafter gi modes),

$$
\begin{aligned}
\left(\frac{\delta \omega_{\mathrm{mag}}}{\omega_{0}}\right)_{g}= & \frac{1}{2} \frac{B_{0}^{2}}{4 \pi \rho_{c} R^{2} \omega_{0}^{2}} \frac{N_{\mathrm{max}}^{2}}{\omega_{0}^{2}} \Lambda_{k, m}(v) C_{k, m}(v) \\
& \times \frac{\int_{x_{t ; i}}^{x_{t ; e}} \frac{b_{\mathrm{r}}^{2} \widehat{N}^{2}}{\left(\rho / \rho_{c}\right) x^{2}} \widehat{N} \frac{\mathrm{d} x}{x}}{\int_{x_{t ; i}}^{x_{t ; e}} \widehat{N} \frac{\mathrm{d} x}{x}},
\end{aligned}
$$

with

$$
C_{k, m}(v)=\frac{\int_{0}^{\pi}\left[H_{\theta}^{2}(\cos \theta)+H_{\varphi}^{2}(\cos \theta)\right] \cos ^{2} \theta \sin \theta \mathrm{d} \theta}{\int_{0}^{\pi}\left[H_{\theta}^{2}(\cos \theta)+H_{\varphi}^{2}(\cos \theta)\right] \sin \theta \mathrm{d} \theta},
$$

where $v=2 \Omega / \omega_{0}$ is the spin parameter and $\Lambda_{k, m}(v)$ is the horizontal eigenvalues of the Hough functions (e.g. Hough 1898; Lee \& Saio 1997; Townsend 2003) $\left\{H_{r}(\cos \theta), H_{\theta}(\cos \theta), H_{\varphi}(\cos \theta)\right\}$ that generalise the spherical harmonics when the rotation within the TAR is taken into 
account (we refer to Appendix A for the details of their definition).

\subsection{Asymptotic $p$ modes}

We can apply the same method to the case of high-frequency acoustic modes, which probe the external layers of stars. In the same way, we isolate using Eq. (12) the dominant terms in Eq. (B.2), which are $\propto \xi_{\mathrm{r}}^{\prime \prime} \xi_{\mathrm{r}}^{*}$

$\left(\frac{\delta \omega_{\mathrm{mag}}}{\omega_{0}}\right)_{p}=\frac{B_{0}^{2}}{8 \pi \omega_{0}^{2}} D_{l, m} \sum_{i=\{\theta, \varphi\}} \frac{\int_{r_{t}}^{R}\left|\left(r b_{i} \xi_{\mathrm{r}}\right)^{\prime}\right|^{2} \mathrm{~d} r}{\int_{r_{t}}^{R}\left|\xi_{\mathrm{r}}\right|^{2} \rho r^{2} \mathrm{~d} r}$,

where $D_{l, m}=\int_{0}^{\pi} \sin ^{2} \theta\left|Y_{l}^{m}\right|^{2} \sin \theta \mathrm{d} \theta$ is computed in Appendix D.3. As for the angular coupling coefficients $C_{l, m}$ computed for asymptotic $g$ modes, we also identify here that $D_{l,-m}=D_{l, m}$. We have introduced the internal turning point $r_{t}=\sqrt{l(l+1)} c / \omega_{0}$ for which $S_{l}\left(r_{t}\right)=\omega_{0}$. In the case of low-degree high-frequency acoustic modes, we have $r_{t} \rightarrow 0$. Using the asymptotic JWKB solution given in Eq. (12) and the theory of rapidly oscillating integrals (see Appendix C), we finally obtain

$\left(\frac{\delta \omega_{\mathrm{mag}}}{\omega_{0}}\right)_{p}=\frac{D_{l, m}}{2} \sum_{i=\{\theta, \varphi\}} \frac{\int_{0}^{R} \frac{\left(V_{\mathrm{A}}^{i}\right)^{2}}{c^{2}} \frac{\mathrm{d} r^{\prime}}{c}}{\int_{0}^{R} \frac{\mathrm{d} r^{\prime}}{c}}$,

where $V_{\mathrm{A}}^{i}=B_{i} / \sqrt{4 \pi \rho}$. We again recover exactly the same form as for the asymptotic rotational splittings, but for the horizontal components of the magnetic field. As in the case of lowfrequency $g$ modes, high-frequency acoustic modes, which have mostly vertical Lagrangian displacements, allow us to probe the orthogonal horizontal (latitudinal and azimuthal) components of the field.

\subsection{Mixed modes}

As in Goupil et al. (2013), we can express the magnetic splittings for mixed (gravito-acoustic) modes using the expressions of the splittings of asymptotic gravity and acoustic modes:

$\left(\frac{\delta \omega_{\mathrm{mag}}}{\omega_{0}}\right)=\left(\frac{\delta \omega_{\mathrm{mag}}}{\omega_{0}}\right)_{g} \zeta+\left(\frac{\delta \omega_{\mathrm{mag}}}{\omega_{0}}\right)_{p}(1-\zeta)$,

where we have introduced the

$\zeta=\frac{I_{g}}{I}=\frac{\int_{r_{t ; i}}^{r_{t, e}}\left(\xi_{\mathrm{r}}^{2}+l(l+1) \xi_{\mathrm{h}}^{2}\right) r^{2} \mathrm{~d} r}{\int_{0}^{R}\left(\xi_{\mathrm{r}}^{2}+l(l+1) \xi_{\mathrm{h}}^{2}\right) r^{2} \mathrm{~d} r}$

function, with $I_{g}$ and $I$ being the inertia of their $g$-dominated component (the so-called $g-m$ modes) and their total inertia, respectively. It quantifies their behaviour as a $g$-dominated mode when $\zeta=1$ or as a $p$-dominated mode (the so-called $p-m$ modes) when $\zeta \rightarrow 0$.

It is interesting here to make the analogy with the case of rotation studied in Goupil et al. (2013). They showed that for the dipolar modes $(l=1)$

$\delta \omega \approx \frac{\zeta}{2}\langle\Omega\rangle_{\text {core }}+(1-\zeta)\langle\Omega\rangle_{\text {envelope }}$, where

$\langle\Omega\rangle_{\text {core }} \approx \frac{1}{2 \sqrt{l(l+1)}}\left(\frac{\omega_{0}}{\Omega_{c}}\right)^{2} \frac{\int_{\text {core }} \Omega(x) N \frac{\mathrm{d} x}{x}}{\int_{\text {core }} N \frac{\mathrm{d} x}{x}}$,

with $\Omega_{c}=\left(G M / R^{3}\right)^{1 / 2}$ the critical Keplerian velocity, and

$\langle\Omega\rangle_{\text {envelope }} \approx \frac{1}{2} \frac{\int_{\text {envelope }} \Omega(x) \frac{\mathrm{d} x}{c}}{\int_{\text {envelope }} \frac{\mathrm{d} x}{c}}$.

Using Eqs. (20) and (24), we identify the analogy between $\Omega$ in the core and $\left(\omega_{\mathrm{A}}^{r}\right)^{2} N^{2} / \omega_{0}^{3}$ in the case of g-dominated modes and between $\Omega$ in the envelope and $\omega_{0}\left(V_{\mathrm{A}}^{i}\right)^{2} / c^{2}$ for $p$-dominated modes. This shows the potential power of mixed modes to probe the magnetism of stars where they propagate from their surfaces to their cores.

\section{Key application: evolved low-mass and intermediate-mass stars}

Because of all the key results they have provided within the theory of stellar evolution and the study of the internal dynamics of stars based on space-based asteroseismology (e.g. Hekker \& Christensen-Dalsgaard 2017; Aerts et al. 2019), we chose here to apply our theoretical results to a typical $1.5 M_{\odot}$ $2.8 \mathrm{Gyr}$ old intermediate-mass red giant star (with a solar metallicity $Z=0.02$ ). The profiles of its Brunt-Vaïsälä and Lamb frequencies for dipolar and quadrupolar modes $\left(N, S_{l=1}, S_{l=2}\right.$, respectively) are represented in Fig. 1 (left panel) where the position of $\nu_{\max }=172.2 \mu \mathrm{Hz}$, the frequency of maximum oscillation power, is reported by the thick horizontal orange line.

First, we computed the topology of a mixed (with both poloidal and toroidal components) relaxed fossil magnetic field in its radiative core following the method introduced in Prat et al. (2019) and the theoretical modelling for this field by Duez \& Mathis (2010). As in Bugnet et al. (2021), the chosen boundary conditions are $b_{\mathrm{r}}=0$ at the boundary of the radiative core and convective envelope. Its amplitude was fixed to $B_{0}=10^{6} \mathrm{G}$ and its geometry is reported in Fig. 1 (right panel). For this possible field amplitude (Cantiello et al. 2016), magnetic frequency splittings should be detectable within Kepler data (Bugnet et al. 2021). The thin black lines represent the poloidal field lines, and the colours give the amplitude of the toroidal component of the field (the boundary of the radiative core is given by the thick black line).

Then, we computed the magnetic splittings with their complete expression $\left(\delta \omega_{\text {mag }} / \omega_{0}\right)_{\text {num }}$ given in Appendix B using the combination of the GYRE and MESA codes (Townsend \& Teitler 2013; Paxton et al. 2011) for the dipolar $(l=1)$ and quadrupolar $(l=2)$ mixed modes (represented by the dots in the left panel (right panel) of Fig. 2). We computed the corresponding asymptotic prediction $\left(\delta \omega_{\text {mag }} / \omega_{0}\right)_{\text {asympt }}$ using Eq. (20) (solid lines in Fig. 2). The complete and asymptotic expressions were compared based on the relative error defined as $\left[\left(\delta \omega_{\text {mag }} / \omega_{0}\right)_{\text {asympt }}-\left(\delta \omega_{\text {mag }} / \omega_{0}\right)_{\text {num }}\right] /\left(\delta \omega_{\text {mag }} / \omega_{0}\right)_{\text {num }}$ (Fig. 2, bottom panels). In the low-frequency regime, the agreement is excellent for g-dominated mixed modes with a relative error 

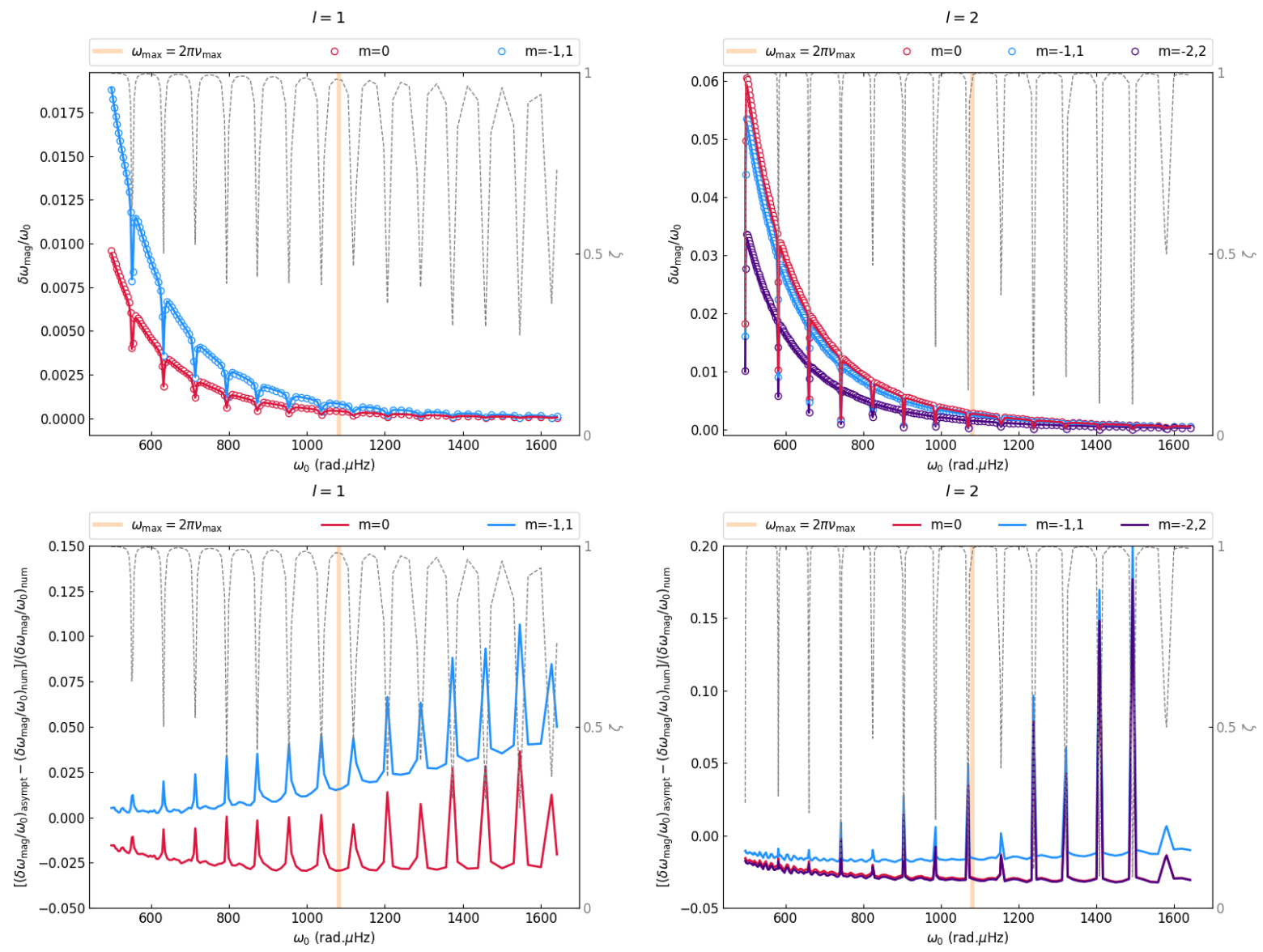

Fig. 2. First-order magnetic splitting for dipolar $(l=1$, left top panel $)$ and quadrupolar $(l=2$, right top panel $)$ mixed modes in the studied $1.5 M_{\odot}$ red giant star. The dots correspond to the full expression for the splitting (Eqs. (B.1) and (B.2)) computed numerically using the GYRE and MESA codes, and the solid lines are the asymptotic solutions given in Eq. (20). The $\zeta$ function related to the mixed character of modes propagating in evolved low-mass stars is plotted as a dotted grey line. Finally, the vertical thick orange line indicates the position of $\omega_{\max }=2 \pi v_{\max }$. Bottom panels: relative errors between the asymptotic magnetic splittings as derived in Eq. (20) and their full expression given in Appendix B for dipolar (left panel) and quadrupolar (right panel) modes, respectively.

lower than $4 \%$ for dipolar modes and $3 \%$ for quadrupolar modes outside the dips of the $\zeta$ function. These results are coherent with the high radial orders of the obtained mixed modes, that is, $n \in[-138,-24]$ for the dipolar modes and $n \in[-244,-56]$ for the quadrupolar modes, for which the JWKB approximation works well. This demonstrates the high potential of using the asymptotic magnetic splittings for intensive seismic modelling where they can be very useful to explore a broad space of stellar parameters (see e.g. the work by Van Beeck et al. 2020, for main-sequence intermediate-mass stars).

As this has already been identified in Eq. (18), the effects of the studied dipolar mixed (poloidal + toroidal) fossil magnetic field on the frequencies of $g$-dominated mixed modes are the same for pro- and retrograde modes. This strongly differs from the case of the frequency splittings induced by the stellar rotation, which are due to the combination of the Coriolis acceleration and of the change in reference frame (e.g. Aerts et al. 2010),

$\delta \omega_{\mathrm{rot}}=-\frac{\left\langle\boldsymbol{\xi}, \boldsymbol{F}_{\boldsymbol{c}}(\boldsymbol{\xi})\right\rangle+\left\langle\boldsymbol{\xi}, \boldsymbol{F}_{\boldsymbol{f}}(\boldsymbol{\xi})\right\rangle}{2 \omega_{0}\langle\boldsymbol{\xi}, \boldsymbol{\xi}\rangle}$,

where $\boldsymbol{F}_{\boldsymbol{c}}\left(\boldsymbol{\xi}_{0}\right)=2 i \omega_{0} \boldsymbol{\Omega} \wedge \boldsymbol{\xi}_{0}$ is the Coriolis acceleration operator and $\boldsymbol{F}_{\boldsymbol{f}}\left(\boldsymbol{\xi}_{0}\right)=-2 m \omega_{0} \Omega \boldsymbol{\xi}_{0}$ is the advection operator due to the change of reference frame. In the case of a radial differential rotation, we have

$\left\langle\boldsymbol{\xi}, \boldsymbol{F}_{\boldsymbol{c}}(\boldsymbol{\xi})\right\rangle=4 m \omega_{0} \int_{0}^{R} \rho r^{2} \Omega(r)\left[\left|\xi_{\mathrm{h}}\right|^{2}+2 \xi_{\mathrm{r}}^{*} \xi_{\mathrm{h}}\right] \mathrm{d} r$

and

$$
\left\langle\boldsymbol{\xi}, \boldsymbol{F}_{f}(\boldsymbol{\xi})\right\rangle=-2 m \omega_{0}\left(\int_{0}^{R} \rho r^{2}\left(\left|\xi_{\mathrm{r}}\right|^{2}+l(l+1)\left|\xi_{\mathrm{h}}\right|^{2}\right) \Omega(r) \mathrm{d} r\right) .
$$

Therefore, $\delta \omega_{\text {rot }}$ is proportional to $m$, leading to opposite rotational frequency splittings for pro- and retrograde modes. This is illustrated in Fig. 3, where we represent in the middle column this rotational splitting when a typical two-zone differential rotation with a solid-body rotation $\Omega_{\text {core }} /(2 \pi)=0.5 \mu \mathrm{Hz}$ in the radiative core and $\Omega_{\text {envelope }} /(2 \pi)=\left(\Omega_{\text {core }} / 10\right) / 2 \pi=$ $0.05 \mu \mathrm{Hz}$ in the convective envelope is assumed (Gehan et al. 2018). When added to the magnetic frequency splitting, which is the same for pro- and retrograde modes, this leads to an asymmetry of the total frequency splitting that scales with the squared amplitude of the field (cf. Eqs. (17) and (20)). This asymmetry will allow asteroseismologists to probe the potential presence of an (axisymmetric) stable fossil magnetic field in the core of evolved low-mass and intermediate-mass stars. We refer to Bugnet et al. (2021) for a detailed characterisation of this 

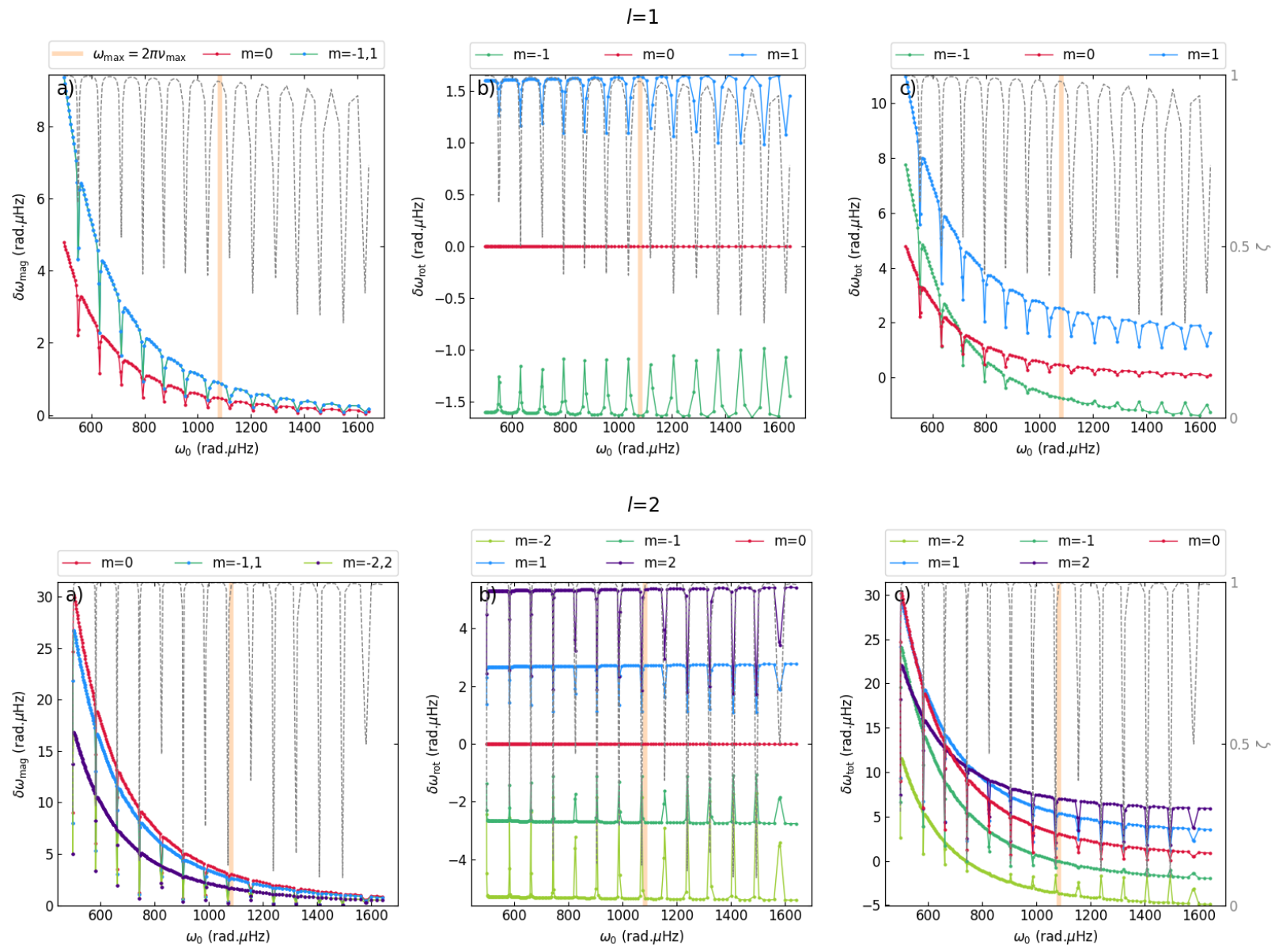

Fig. 3. Leading-order perturbations on the frequencies of dipolar $(l=1$, top panels $)$ and quadripolar $(l=2$, bottom panels $)$ mixed modes in the studied $1.5 M_{\odot}$ red giant star. Left panels: magnetic angular frequency splitting $\delta \omega_{\operatorname{mag}}$ (for $B_{0}=1 \mathrm{MG}$ ) of the $m \in[-l, l]$ modes. Middle panels: angular frequency splitting $\delta \omega_{\text {rot }}$ for a plausible two-layer differential rotation (with $\Omega_{\text {core }}=0.5 \mu \mathrm{Hz}$ and $\Omega_{\text {env }}=\Omega_{\text {core }} / 10$ ) of the same mixed modes is represented. Right panels: combination of magnetic and rotational splittings of mixed-mode angular frequencies $\delta \omega_{\text {tot }}=\delta \omega_{\text {mag }}+\delta \omega_{\text {rot }}$. The vertical orange line indicates in each panel the expected frequency of maximum power of the modes $v_{\max }$. The $\zeta$ function is represented in each case by the grey dashed line.

asymmetry in seismic data. We did not consider the case of $p$-dominated modes during the subgiant phase because Bugnet et al. (2021) demonstrated that magnetic signatures would not be detectable for them.

\section{Conclusion and perspectives}

We have established the asymptotic values of the magnetic splittings of low-frequency gravity and gravito-inertial (gi) modes and high-frequency acoustic modes. These results were applied to the case of mixed gravito-acoustic modes such as those that propagate in evolved low- and intermediate-mass red giant stars. They were derived in the case of a large-scale axisymmetric mixed (poloidal + toroidal) dipolar field that can be representative of a stable fossil field or of the large-scale axisymmetric dipolar component of a dynamo-generated field. The main results that we obtained are listed below.

The expressions obtained for the magnetic splittings in the asymptotic limits of low-frequency $g$ ( $g i$ ) modes and highfrequency $p$ modes are very similar in their form to those of rotational splittings in the same limits. This opens the path to potential inversions of the internal distribution of magnetic fields in stellar interiors when these signatures can be detected in stellar oscillation frequency spectra.
For asymptotic $g(g i)$ modes, the splittings scale as the normalised squared ratio of the local Alfvén frequency to the mode frequency integrated along the buoyancy radius. For asymptotic $p$ modes, they scale as the normalised squared ratio of the Alfvén velocity to the sound speed integrated along the acoustic path.

In the case of the dipolar configuration we studied, magnetic frequency splittings are the same for pro- and retrograde modes ( $m>0$ and $m<0$, respectively). This allows us to distinguish them from rotational frequency splittings that have opposite values for pro- and retrograde modes. This leads to an asymmetry of the total frequency splittings when the two effects are added. It could be detectable within high-precision asteroseismic data for sufficient field amplitude. We refer to Bugnet et al. (2021) for a complete seismic characterisation of this asymmetry and for the study of this required amplitude in the evolution of low-mass and intermediate-mass stars.

In the case of the large-scale mixed dipolar configuration, which is treated here, low-frequency $g$ ( $g i)$ modes and highfrequency $p$ modes explore different components of the magnetic field because of the form of the linearised Lorentz force, which involves $\boldsymbol{\xi} \wedge \boldsymbol{B}$ terms. On the one hand, mostly horizontal lowfrequency $g(g i)$ modes, which propagate in the central regions of stars, probe the orthogonal radial component of the field. On the other hand, mostly vertical high-frequency $p$ modes, which 


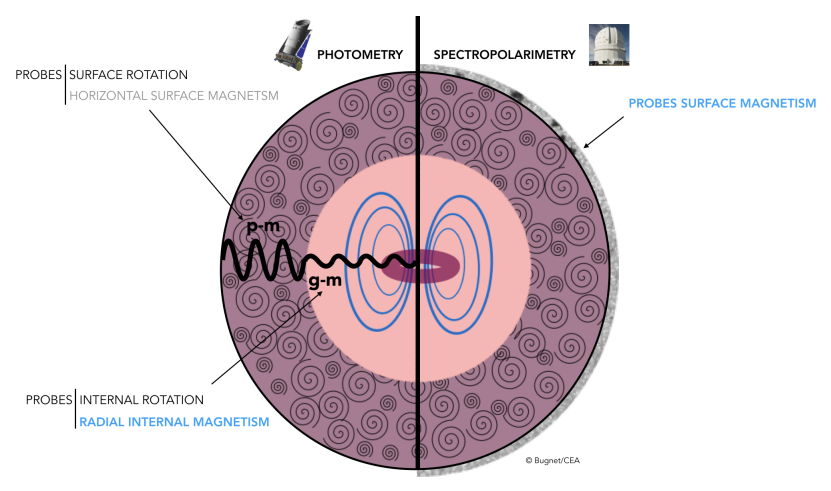

Fig. 4. Global strategy for probing the internal magnetism of stars using asteroseismology (in potential synergy with spectropolarimetry). Here, the scheme is designed for evolved low- and intermediate-mass stars.

propagate in the external regions of stars, probe the orthogonal horizontal (latitudinal and azimuthal) components of the field. As in the case of the rotation, where mixed modes have allowed us to probe the rotation of the core and of the external envelope with their $g$-dominated $(g-m)$ and $p$-dominated $(p-m)$ components, respectively, this opens the path to probe the stellar magnetic field from the surface to the core of stars. Because high frequencies of asymptotic $p$ modes will be more distant than low frequencies of asymptotic $g$ modes from the Alfvén frequency, the seismic signatures of the external field can be more difficult to detect in the case of a low-amplitude field. This can be fixed in the case of bright stars such as those observed by the Transiting Exoplanet Survey Satellite (TESS; Ricker et al. 2015), and in the future by the PLAnetary Transits and Oscillations of stars (PLATO) mission (Rauer et al. 2014), by combining spectropolarimetic observations to determine the configuration and the strength of the field at the surface and seismic signatures to probe the core magnetism. This strategy is summarised in Fig. 4.

In a near future, the derived asymptotic relations should be tested and systematically used for all classes of pulsators. In addition, more complex (non-dipolar and non-axisymmetric) magnetic topologies must be studied (e.g. Prat et al. 2020). This should allow us to build a complete knowledge of the magnetism of stars from their surfaces to their cores, step by step.

Acknowledgements. The authors thank the referee and Pr. J. ChristensenDalsgaard for their very constructive comments and remarks that allowed us to improve the article. St. M., L. B., V. P., and K. A. acknowledge support from the European Research Council through ERC grant SPIRE 647383. All the members from CEA acknowledge support from GOLF and PLATO CNES grants of the Astrophysics Division at CEA. S. Mathur acknowledges support by the Ramon y Cajal fellowship number RYC-2015-17697. We made great use of the megyr python package for interfacing MESA and GYRE codes.

\section{References}

Abramowitz, M., \& Stegun, I. A. 1972, Handbook of Mathematical Functions (New York: Dover Publications, INC.)

Aerts, C. 2021, Rev. Mod. Phys., 93, 015001

Aerts, C., Christensen-Dalsgaard, J., \& Kurtz, D. W. 2010, Asteroseismology (Springer)

Aerts, C., Mathis, S., \& Rogers, T. M. 2019, ARA\&A, 57, 35

Aurière, M., Konstantinova-Antova, R., Charbonnel, C., et al. 2015, A\&A, 574, A90

Beck, P. G., Montalban, J., Kallinger, T., et al. 2012, Nature, 481, 55

Benomar, O., Takata, M., Shibahashi, H., Ceillier, T., \& García, R. A. 2015, MNRAS, 452, 2654

Bouabid, M. P., Dupret, M. A., Salmon, S., et al. 2013, MNRAS, 429, 2500
Bugnet, L., Prat, V., Mathis, S., et al. 2021, A\&A, submitted [arXiv:2102.01216]

Braithwaite, J., \& Spruit, H. C. 2004, Nature, 431, 819

Braithwaite, J., \& Spruit, H. C. 2017, R. Soc. Open Sci., 4, 16027

Brun, A. S., \& Browning, M. K. 2017, Liv. Rev. Sol. Phys., 14, 4

Cantiello, M., Mankovich, C., Bildsten, L., Christensen-Dalsgaard, J., \& Paxton, B. 2014, ApJ, 788, 93

Cantiello, M., Fuller, J., \& Bildsten, L. 2016, ApJ, 824, 14

Ceillier, T., Eggenberger, P., García, R. A., \& Mathis, S. 2013, A\&A, 555, A54

Christensen-Dalsgaard, J. 2015, From the Sun to the distant stars, and back Future research directions (Cambridge University Press), 276

Christophe, S., Ballot, J., Ouazzani, R. M., Antoci, V., \& Salmon, S. J. A. J. 2018, A\&A, 618, A47

Couvidat, S., García, R. A., Turck-Chièze, S., et al. 2003, ApJ, 597, L77

Deheuvels, S., García, R. A., Chaplin, W. J., et al. 2012, ApJ, 756, 19

Deheuvels, S., Doğan, G., Goupil, M. J., et al. 2014, A\&A, 564, A27

Deheuvels, S., Ballot, J., Beck, P. G., et al. 2015, A\&A, 580, A96

Deheuvels, S., Ballot, J., Eggenberger, P., et al. 2020, A\&A, 641, A117

Domingo, V., Fleck, B., \& Poland, A. I. 1995, Sol. Phys., 162, 1

Donati, J. F., \& Landstreet, J. D. 2009, ARA\&A, 47, 333

Duez, V., \& Mathis, S. 2010, A\&A, 517, A58

Eckart, C. 1960, Hydrodynamics of Oceans and Atmospheres (Pergamon Press)

Eggenberger, P., Montalbán, J., \& Miglio, A. 2012, A\&A, 544, L4

Erdelyi, A. 1956, Asymptotic Expansions (Dover Publications, Inc.)

Fröman, N., \& Fröman, P. O. 2005, Physical Problems Solved by the PhaseIntegral Method (North-Holland Publishing Company)

Fuller, J., Piro, A. L., \& Jermyn, A. S. 2019, MNRAS, 485, 3661

Gabriel, A. H., Grec, G., Charra, J., et al. 1995, Sol. Phys., 162, 61

García, R. A., \& Ballot, J. 2019, Liv. Rev. Sol. Phys., 16, 4

García, R. A., Turck-Chièze, S., Jiménez-Reyes, S. J., et al. 2007, Science, 316, 1591

Gehan, C., Mosser, B., Michel, E., Samadi, R., \& Kallinger, T. 2018, A\&A, 616, A24

Goode, P. R., \& Thompson, M. J. 1992, ApJ, 395, 307

Gough, D. O. 1993, Astrophysical Fluid Dynamics - Les Houches 1987, 399

Goupil, M. J., Mosser, B., Marques, J. P., et al. 2013, A\&A, 549, A75

Hasan, S. S., Zahn, J. P., \& Christensen-Dalsgaard, J. 2005, A\&A, 444, L29

Hekker, S., \& Christensen-Dalsgaard, J. 2017, A\&ARv, 25, 1

Hough, S. S. 1898, Philos. Trans. R. Soc. London Ser. A, 191, 139

Kiefer, R., \& Roth, M. 2018, ApJ, 854, 74

Lee, U., \& Saio, H. 1997, ApJ, 491, 839

Li, G., Bedding, T. R., Murphy, S. J., et al. 2019, MNRAS, 482, 1757

Li, G., Van Reeth, T., Bedding, T. R., et al. 2020, MNRAS, 491, 3586

Marques, J. P., Goupil, M. J., Lebreton, Y., et al. 2013, A\&A, 549, A74

Mathis, S. 2009, A\&A, 506, 811

Mathis, S., \& Prat, V. 2019, A\&A, 631, A26

Mathis, S., \& Zahn, J. P. 2005, A\&A, 440, 653

Mathur, S., Eff-Darwich, A., García, R. A., \& Turck-Chièze, S. 2008, A\&A, 484, 517

Mestel, L., \& Weiss, N. O. 1987, MNRAS, 226, 123

Mosser, B., Goupil, M. J., Belkacem, K., et al. 2012, A\&A, 548, A10

Ouazzani, R. M., Marques, J. P., Goupil, M. J., et al. 2019, A\&A, 626, A121

Ouazzani, R. M., Lignières, F., Dupret, M. A., et al. 2020, A\&A, 640, A49

Paxton, B., Bildsten, L., Dotter, A., et al. 2011, ApJS, 192, 3

Prat, V., Mathis, S., Buysschaert, B., et al. 2019, A\&A, 627, A64

Prat, V., Mathis, S., Neiner, C., et al. 2020, A\&A, 636, A100

Rashba, T. I., Semikoz, V. B., Turck-Chièze, S., \& Valle, J. W. F. 2007, MNRAS, 377,453

Rauer, H., Catala, C., Aerts, C., et al. 2014, Exp. Astron., 38, 249

Ricker, G. R., Winn, J. N., Vanderspek, R., et al. 2015, J. Astron. Telescopes Instrum. Syst., 1, 014003

Samadi, R., Belkacem, K., \& Sonoi, T. 2015, EAS Publ. Ser., 73-74, 111

Scherrer, P. H., Bogart, R. S., Bush, R. I., et al. 1995, Sol. Phys., 162, 129

Shibahashi, H. 1979, PASJ, 31, 87

Spada, F., Gellert, M., Arlt, R., \& Deheuvels, S. 2016, A\&A, 589, A23

Spruit, H. C. 2002, A\&A, 381, 923

Takata, M., \& Shibahashi, H. 1994, PASJ, 46, 301

Thompson, M. J., Toomre, J., Anderson, E. R., et al. 1996, Science, 272, 1300

Townsend, R. H. D. 2003, MNRAS, 340, 1020

Townsend, R. H. D., \& Teitler, S. A. 2013, MNRAS, 435, 3406

Van Beeck, J., Prat, V., Van Reeth, T., et al. 2020, A\&A, 638, A149

Van Reeth, T., Tkachenko, A., \& Aerts, C. 2016, A\&A, 593, A120

Van Reeth, T., Mombarg, J. S. G., Mathis, S., et al. 2018, A\&A, 618, A24

Varshalovich, D. A., Moskalev, A. N., \& Khersonskii, V. 1988, Quantum Theory of Angular Momentum (World Scientific)

Wade, G. A., Neiner, C., Alecian, E., et al. 2016, MNRAS, 456, 2 
S. Mathis et al.: Probing the internal magnetism of stars using asymptotic magneto-asteroseismology

\section{Appendix A: Hough functions}

The radial Hough functions (Hough 1898) are defined by $H_{r}(\cos \theta)=f(\mu=\cos \theta)$, where $f$ is the solution of the so-called tidal Laplace equation,

$$
\begin{aligned}
& \frac{1-\mu^{2}}{1-v^{2} \mu^{2}} \frac{\mathrm{d}^{2} f}{\mathrm{~d} \mu^{2}}-\frac{2 \mu\left(1-v^{2}\right)}{\left(1-v^{2} \mu^{2}\right)^{2}} \frac{\mathrm{d} f}{\mathrm{~d} \mu} \\
& +\left[\frac{m v\left(1+v^{2} \mu^{2}\right)}{\left(1-v^{2} \mu^{2}\right)^{2}}-\frac{m^{2}}{\left(1-\mu^{2}\right)\left(1-v^{2} \mu^{2}\right)}\right] f \\
& =-\Lambda(v) f,
\end{aligned}
$$

where $v=2 \Omega / \omega$ is the spin factor. In the non-rotating case $(v=$ 0 ), the eigenvalue $\Lambda$ (they are generally indexed by an integer $k$ for a given $m$; see e.g. Lee \& Saio 1997) reduces to $l(l+1)$, where $l$ is the angular degree of the mode, and $H_{r}$ simplifies to the classical associated Legendre polynomial $P_{l}^{m}(\cos \theta)$.

The latitudinal and azimuthal Hough functions are respectively derived,

$H_{\theta}(\cos \theta) \sin \theta=\frac{H_{r}^{\prime} \sin \theta-m v H_{r} \cos \theta}{1-v^{2} \cos ^{2} \theta}$

and

$H_{\varphi}(\cos \theta) \sin \theta=\frac{m H_{r}-v H_{r}^{\prime} \sin \theta \cos \theta}{1-v^{2} \cos ^{2} \theta}$,

where' is the total latitudinal derivative. $H_{\varphi}$ has the same parity as $H_{r}$ with respect to $\theta=\pi / 2$, while $H_{\theta}$ has the opposite parity. In the non-rotating case $(v=0), H_{\theta}$ and $H_{\varphi}$ reduce to $\mathrm{d} P_{l}^{m} / \mathrm{d} \theta$ and $m P_{l}^{m} / \sin \theta$, respectively.

\section{Appendix B: General expression of magnetic splittings}

We recall all the non-zero-average terms of the work of the Lorentz force $\mu_{0} \delta \boldsymbol{F}_{\mathrm{L}} \cdot \boldsymbol{\xi}^{*}$. All these terms involve either only poloidal components of the magnetic field or only the toroidal component, and they are grouped accordingly as in Prat et al (2019). All terms are composed of a purely radial part multiplied by a purely latitudinal part, and the prime symbol $\left({ }^{\prime}\right)$ is a total radial or latitudinal derivative, depending on the considered part.

\section{B.1. Poloidal terms}

We define $A=\left[\left(r b_{\theta}\right)^{\prime}+b_{\mathrm{r}}\right]$. The terms that involve poloidal components are obtained,

$$
\begin{aligned}
- & m \frac{\xi_{\mathrm{h}} b_{\theta} A \xi_{\mathrm{r}}^{*}}{r^{2}} H_{\mathrm{r}} H_{\varphi} \sin \theta+\frac{\left(r \xi_{\mathrm{r}} b_{\theta}\right)^{\prime} A \xi_{\mathrm{r}}^{*}}{r^{2}} H_{\mathrm{r}}^{2} \sin ^{2} \theta \\
& -\frac{\left(r \xi_{\mathrm{h}} b_{\mathrm{r}}\right)^{\prime} A \xi_{\mathrm{r}}^{*}}{r^{2}} H_{\mathrm{r}} H_{\theta} \sin \theta \cos \theta+\frac{\xi_{\mathrm{r}} b_{\theta} A \xi_{\mathrm{h}}^{*}}{r^{2}} H_{\theta}\left(H_{\mathrm{r}} \sin ^{2} \theta\right)^{\prime} \\
& -\frac{A b_{\mathrm{r}}\left|\xi_{\mathrm{h}}\right|^{2}}{r^{2}} H_{\theta}\left(H_{\theta} \sin \theta \cos \theta\right)^{\prime}+m \frac{A b_{\mathrm{r}}\left|\xi_{\mathrm{h}}\right|^{2}}{r^{2}} H_{\theta} H_{\varphi} \cos \theta \\
& -m \frac{\left(\xi_{\mathrm{h}} b_{\theta}\right)^{\prime} b_{\theta} \xi_{\mathrm{r}}^{*}}{r} H_{\mathrm{r}} H_{\varphi} \sin \theta+\frac{\left(r \xi_{\mathrm{r}} b_{\theta}\right)^{\prime \prime} b_{\theta} \xi_{\mathrm{r}}^{*}}{r} H_{\mathrm{r}}^{2} \sin ^{2} \theta \\
& -\frac{\left(r \xi_{\mathrm{h}} b_{\mathrm{r}}\right)^{\prime \prime} b_{\theta} \xi_{\mathrm{r}}^{*}}{r} H_{\mathrm{r}} H_{\theta} \sin \theta \cos \theta+\frac{b_{\theta}^{2}\left|\xi_{\mathrm{r}}\right|^{2}}{r^{2}} H_{\mathrm{r}} \sin \theta\left[\frac{\left(H_{\mathrm{r}} \sin ^{2} \theta\right)^{\prime}}{\sin \theta}\right]^{\prime} \\
& -\frac{\xi_{\mathrm{h}} b_{\mathrm{r}} b_{\theta} \xi_{\mathrm{r}}^{*}}{r^{2}} H_{\mathrm{r}} \sin \theta\left[\frac{\left(H_{\theta} \sin \theta \cos \theta\right)^{\prime}}{\sin \theta}\right]^{\prime} \\
& +m \frac{\xi_{\mathrm{h}} b_{\mathrm{r}} b_{\theta} \xi_{\mathrm{r}}^{*}}{r^{2}} H_{\mathrm{r}} \sin \theta\left(H_{\varphi} \frac{\cos \theta}{\sin \theta}\right)^{\prime}+m \frac{\left(\xi_{\mathrm{h}} b_{\theta}\right)^{\prime} b_{\mathrm{r}} \xi_{\mathrm{h}}^{*}}{r} H_{\theta} H_{\varphi} \cos \theta
\end{aligned}
$$

$$
\begin{aligned}
& -\frac{\left(r \xi_{\mathrm{r}} b_{\theta}\right)^{\prime \prime} b_{\mathrm{r}} \xi_{\mathrm{h}}^{*}}{r} H_{\mathrm{r}} H_{\theta} \sin \theta \cos \theta+\frac{\left(r \xi_{\mathrm{h}} b_{\mathrm{r}}\right)^{\prime \prime} b_{\mathrm{r}} \xi_{\mathrm{h}}^{*}}{r} H_{\theta}^{2} \cos ^{2} \theta \\
& -\frac{\xi_{\mathrm{r}} b_{\mathrm{r}} b_{\theta} \xi_{\mathrm{h}}^{*}}{r^{2}} H_{\theta} \cos \theta\left[\frac{\left(H_{\mathrm{r}} \sin ^{2} \theta\right)^{\prime}}{\sin \theta}\right]^{\prime} \\
& +\frac{b_{\mathrm{r}}^{2}\left|\xi_{\mathrm{h}}\right|^{2}}{r^{2}} H_{\theta} \cos \theta\left[\frac{\left(H_{\theta} \sin \theta \cos \theta\right)^{\prime}}{\sin \theta}\right]^{\prime} \\
& -m \frac{b_{\mathrm{r}}^{2}\left|\xi_{\mathrm{h}}\right|^{2}}{r^{2}} H_{\theta} \cos \theta\left(H_{\varphi} \frac{\cos \theta}{\sin \theta}\right)^{\prime}+\frac{\left(r \xi_{\mathrm{h}} b_{\mathrm{r}}\right)^{\prime} b_{\theta} \xi_{\mathrm{h}}^{*}}{r^{2}} H_{\varphi}\left(H_{\varphi} \sin \theta \cos \theta\right)^{\prime} \\
& +\frac{b_{\theta}^{2}\left|\xi_{\mathrm{h}}\right|^{2}}{r^{2}} H_{\varphi}\left[\sin \theta\left(H_{\varphi} \sin \theta\right)^{\prime}\right]^{\prime}-m^{2} \frac{b_{\theta}^{2}\left|\xi_{\mathrm{h}}\right|^{2}}{r^{2}} H_{\varphi}^{2} \\
& +m \frac{\left(r \xi_{\mathrm{r}} b_{\theta}\right)^{\prime} b_{\theta} \xi_{\mathrm{h}}^{*}}{r^{2}} H_{\mathrm{r}} H_{\varphi} \sin \theta-m \frac{\left(r \xi_{\mathrm{h}} b_{\mathrm{r}}\right)^{\prime} b_{\theta} \xi_{\mathrm{h}}^{*}}{r^{2}} H_{\theta} H_{\varphi} \cos \theta \\
& -m \frac{\xi_{\mathrm{r}} b_{\theta} b_{\mathrm{r}} \xi_{\mathrm{h}}^{*}}{r^{2}} H_{\varphi} \frac{\cos \theta}{\sin ^{2} \theta}\left(H_{\mathrm{r}} \sin ^{2} \theta\right)^{\prime}+m \frac{b_{\mathrm{r}}^{2}\left|\xi_{\mathrm{h}}\right|^{2}}{r^{2}} H_{\varphi} \frac{\cos \theta}{\sin ^{2} \theta}\left(H_{\theta} \sin \theta \cos \theta\right)^{\prime} \\
& -m^{2} \frac{b_{\mathrm{r}}^{2}\left|\xi_{\mathrm{h}}\right|^{2}}{r^{2}} H_{\varphi}^{2} \frac{\cos ^{2} \theta}{\sin ^{2} \theta}+\frac{\left(r \xi_{\mathrm{h}} b_{\mathrm{r}}\right)^{\prime \prime} b_{\mathrm{r}} \xi_{\mathrm{h}}^{*}}{r} H_{\varphi}^{2} \cos ^{2} \theta \\
& +\frac{\left(\xi_{\mathrm{h}} b_{\theta}\right)^{\prime} b_{\mathrm{r}} \xi_{\mathrm{h}}^{*}}{r} H_{\varphi} \cos \theta\left(H_{\varphi} \sin \theta\right)^{\prime} \text {. }
\end{aligned}
$$

\section{B.2. Toroidal terms}

The terms involving the toroidal component are derived,

$$
\begin{aligned}
& 2 \frac{\left(r \xi_{\mathrm{r}} b_{\varphi}\right)^{\prime} b_{\varphi} \xi_{\mathrm{h}}^{*}}{r^{2}} H_{\mathrm{r}} H_{\theta} \sin \theta \cos \theta+2 \frac{b_{\varphi}^{2}\left|\xi_{\mathrm{h}}\right|^{2}}{r^{2}} H_{\theta} \cos \theta\left(H_{\theta} \sin \theta\right)^{\prime} \\
& +2 m \frac{b_{\varphi}^{2}\left|\xi_{\mathrm{h}}\right|^{2}}{r^{2}} H_{\theta} H_{\varphi} \cos \theta+\frac{\left(r \xi_{\mathrm{r}} b_{\varphi}\right)^{\prime \prime} b_{\varphi} \xi_{\mathrm{r}}^{*}}{r} H_{\mathrm{r}}^{2} \sin ^{2} \theta \\
& +\frac{\left(\xi_{\mathrm{h}} b_{\varphi}\right)^{\prime} b_{\varphi} \xi_{\mathrm{r}}^{*}}{r} H_{\mathrm{r}} \sin \theta\left(H_{\theta} \sin \theta\right)^{\prime}+\frac{\left(r \xi_{\mathrm{r}} b_{\varphi}\right)^{\prime} b_{\varphi} \xi_{\mathrm{h}}^{*}}{r^{2}} H_{\theta}\left(H_{\mathrm{r}} \sin ^{2} \theta\right)^{\prime} \\
& \quad+\frac{b_{\varphi}^{2}\left|\xi_{\mathrm{h}}\right|^{2}}{r^{2}} H_{\theta}\left[\sin \theta\left(H_{\theta} \sin \theta\right)^{\prime}\right]^{\prime}-m^{2} \frac{b_{\varphi}^{2}\left|\xi_{\mathrm{h}}\right|^{2}}{r^{2}} H_{\theta}^{2} \\
& +\frac{\left(r \xi_{\mathrm{r}} b_{\varphi}\right)^{\prime}\left(r b_{\varphi}\right)^{\prime} \xi_{\mathrm{r}}^{*}}{r^{2}} H_{\mathrm{r}}^{2} \sin { }^{2} \theta+\frac{\xi_{\mathrm{h}} b_{\varphi}\left(r b_{\varphi}\right)^{\prime} \xi_{\mathrm{r}}^{*}}{r^{2}} H_{\mathrm{r}} \sin \theta\left(H_{\theta} \sin \theta\right)^{\prime} \\
& \quad+m \frac{\xi_{\mathrm{r}} b_{\varphi}\left(r b_{\varphi}\right)^{\prime} \xi_{\mathrm{h}}^{*}}{r^{2}} H_{\mathrm{r}} H_{\varphi} \sin \theta-m^{2} \frac{b_{\varphi}^{2}\left|\xi_{\mathrm{r}}\right|^{2}}{r^{2}} H_{\mathrm{r}}^{2} .
\end{aligned}
$$

\section{Appendix C: JWKB method for ordinary differential equations and rapidly oscillating integrals}

On the one hand, studying the dynamics of asymptotic lowfrequency $g$ and high-frequency $p$ modes implies solving Schrödinger-like equations of the form

$\frac{\mathrm{d}^{2} \Psi(x)}{\mathrm{d} x^{2}}+\lambda^{2} \hat{k}_{x}^{2}(x) \Psi(x)=0$,

where $\lambda$ is a large parameter and $\hat{k}_{x}$ is a normalised vertical wave number. Applying the JWKB (for Jeffreys-Wentzel-KrammerBrillouin) method leads for $\lambda \gg 1$ to the following solution (e.g. Erdelyi 1956; Fröman \& Fröman 2005):

$$
\begin{aligned}
\Psi(x)= & \frac{1}{\sqrt{\hat{k}_{x}(x)}}\left[A_{+} \exp \left(i \lambda \int^{x} \hat{k}_{x}\left(x^{\prime}\right) \mathrm{d} x^{\prime}\right)\right. \\
& \left.+A_{-} \exp \left(-i \lambda \int^{x} \hat{k}_{x}\left(x^{\prime}\right) \mathrm{d} x^{\prime}\right)\right] .
\end{aligned}
$$

On the other hand, we have to compute integrals of the form

$I=\int_{a}^{b} g(x) \exp [i \lambda f(x)] \mathrm{d} x$ 
to calculate their magnetic splittings. In the cases where $\lambda \gg 1$, they can be approximated by

$I \rightarrow \lambda \rightarrow \infty \sum_{\left|x_{i}\right|} g\left(x_{i}\right) \sqrt{\frac{2 \pi}{\left|f^{\prime \prime}\left(x_{i}\right)\right|}} \exp \left[i \lambda\left(f\left(x_{i}\right)-\frac{\pi}{4} \operatorname{sgn}\left(f^{\prime \prime}\left(x_{i}\right)\right)\right)\right]$,

where the $x_{i}$ are the points such that $f^{\prime}\left(x_{i}\right)=0$ (Erdelyi 1956). If $f(x)=\int^{x} \hat{k}_{x}\left(x^{\prime}\right) \mathrm{d} x^{\prime}$, the $x_{i}$ are the turning points such that $\left(\hat{k}_{x}\left(x_{i}\right)\right)^{2}=0$.

\section{Appendix D: Algebra for computing horizontal integrals}

\section{D.1. Spherical harmonics}

\section{D.1.1. Definitions}

The spherical harmonics are defined by (e.g. Varshalovich et al. 1988)

$Y_{l}^{m}(\theta, \varphi)=\mathcal{N}_{l}^{m} P_{l}^{|m|}(\cos \theta) e^{i m \varphi}$,

where $P_{l}^{|m|}(\cos \theta)$ is the associated Legendre function, and $\mathcal{N}_{l}^{m}$ the normalisation coefficient

$\mathcal{N}_{l}^{m}=(-1)^{\frac{(m+|m|)}{2}}\left[\frac{2 l+1}{4 \pi} \frac{(l-|m|) !}{(l+|m|) !}\right]^{\frac{1}{2}}$.

They obey the orthogonality relation,

$\int_{\Omega}\left(Y_{l_{1}}^{m_{1}}(\theta, \varphi)\right)^{*} Y_{l_{2}}^{m_{2}}(\theta, \varphi) \mathrm{d} \Omega=\delta_{l_{1}, l_{2}} \delta_{m_{1}, m_{2}}$,

where $\mathrm{d} \Omega=\sin \theta \mathrm{d} \theta \mathrm{d} \varphi$ and where the complex conjugate spherical harmonic is given by

$\left(Y_{l}^{m}(\theta, \varphi)\right)^{*}=(-1)^{m} Y_{l}^{-m}(\theta, \varphi)$.

\section{D.1.2. Linear recurrence relations}

We introduce useful linear recurrence relations that should be used to analytically compute the $C_{l, m}$ and $D_{l, m}$ coefficients. Using again Varshalovich et al. (1988), we have on the one hand

$\cos \theta Y_{l}^{m}=\alpha_{l, m} Y_{l+1}^{m}+\beta_{l, m} Y_{l-1}^{m}$,

where

$\alpha_{l, m}=\left[\frac{(l+1+m)(l+1-m)}{(2 l+1)(2 l+3)}\right]^{1 / 2}$

and

$\beta_{l, m}=\left[\frac{(l+m)(l-m)}{(2 l+1)(2 l-1)}\right]^{1 / 2}$.

On the other hand, we obtain

$\partial_{\theta} Y_{l}^{m}=A_{l, m} Y_{l}^{m+1} e^{-i \varphi}-B_{l, m} Y_{l}^{m-1} e^{i \varphi}$

$-m \frac{\cos \theta}{\sin \theta} Y_{l}^{m}=A_{l, m} Y_{l}^{m+1} e^{-i \varphi}+B_{l, m} Y_{l}^{m-1} e^{i \varphi}$,

where

$A_{l, m}=\frac{1}{2} \sqrt{[l(l+1)-m(m+1)]}$

and

$B_{l, m}=\frac{1}{2} \sqrt{[l(l+1)-m(m-1)]}$.

\section{D.1.3. Products of spherical functions and specific integrals}

Integrals involving products of spherical harmonics should also be computed to calculate $C_{l, m}$ and $D_{l, m}$. Using the normalisation and the orthogonality of spherical harmonics (Eqs. (D.2)-(D.3)) and their complex conjugate (Eq. (D.4)), we can write

$$
\begin{aligned}
Y_{l_{1}}^{m_{1}}(\theta, \varphi) Y_{l_{2}}^{m_{2}}(\theta, \varphi) & =\sum_{l=\left|l_{1}-l_{2}\right|}^{l_{1}+l_{2}} c_{l_{1}, m_{1}, l_{2}, m_{2}}^{l} Y_{l}^{m_{1}+m_{2}}(\theta, \varphi) \\
& =(-1)^{\left(m_{1}+m_{2}\right)} \sum_{l=\left|l_{1}-l_{2}\right|}^{l_{1}+l_{2}} \mathcal{I}_{l_{1}, l_{2}, l}^{m_{1}, m_{2},-\left(m_{1}+m_{2}\right)} Y_{l}^{m_{1}+m_{2}}(\theta, \varphi),
\end{aligned}
$$

where we define the integral $\mathcal{I}_{l_{1}, l_{2}, l}^{m_{1}, m_{2}, m}$ like in Varshalovich et al. (1988),

$$
\begin{aligned}
& \mathcal{I}_{l_{1}, l_{2}, l}^{m_{1}, m_{2}, m}=\int_{\Omega} Y_{l_{1}}^{m_{1}}(\theta, \varphi) Y_{l_{2}}^{m_{2}}(\theta, \varphi) Y_{l}^{m}(\theta, \varphi) \mathrm{d} \Omega \\
& \quad=\sqrt{\frac{\left(2 l_{1}+1\right)\left(2 l_{2}+1\right)(2 l+1)}{4 \pi}}\left(\begin{array}{ccc}
l_{1} & l_{2} & l \\
m_{1} & m_{2} & m
\end{array}\right)\left(\begin{array}{ccc}
l_{1} & l_{2} & l \\
0 & 0 & 0
\end{array}\right)
\end{aligned}
$$

with the $3 \mathrm{j}$-Wigner coefficients $(\cdots)$ that are related to the classical Clebsch-Gordan coefficients by

$\left(\begin{array}{ccc}l_{1} & l_{2} & l \\ m_{1} & m_{2} & m\end{array}\right)=\frac{(-1)^{l_{1}-l_{2}-m}}{\sqrt{2 l+1}} C_{l_{1}, m_{1}, l_{2}, m_{2}}^{l,-m}$.

Then, using the initial definition of spherical harmonics (cf. Eqs. (D.1)-(D.2)), we deduce the expansion for the product of two associated Legendre functions,

$P_{l_{1}}^{m_{1}}(\cos \theta) P_{l_{2}}^{m_{2}}(\cos \theta)=\sum_{l=\left|l_{1}-l_{2}\right|}^{l_{1}+l_{2}} d_{l_{1}, m_{1}, l_{2}, m_{2}}^{l} P_{l}^{m_{1}+m_{2}}(\cos \theta)$,

where

$$
\begin{aligned}
d_{l_{1}, m_{1}, l_{2}, m_{2}}^{l}= & (-1)^{\left(m_{1}+m_{2}\right)}(2 l+1) \\
& \times \sqrt{\frac{\left(l_{1}+m_{1}\right) !\left(l_{2}+m_{2}\right) !\left(l-\left(m_{1}+m_{2}\right)\right) !}{\left(l_{1}-m_{1}\right) !\left(l_{2}-m_{2}\right) !\left(l+\left(m_{1}+m_{2}\right)\right) !}} \\
& \times\left(\begin{array}{ccc}
l_{1} & l_{2} & l \\
m_{1} & m_{2} & -\left(m_{1}+m_{2}\right)
\end{array}\right)\left(\begin{array}{ccc}
l_{1} & l_{2} & l \\
0 & 0 & 0
\end{array}\right) .
\end{aligned}
$$

The last relevant integral is

$$
\begin{aligned}
\int_{-1}^{1} P_{l}^{m}(\mu) \mathrm{d} \mu= & \frac{(-1)^{l}+(-1)^{m}}{\left(\frac{l-m}{2}\right) ! \Gamma\left(\frac{l+3}{2}\right)} \\
& \times 2^{m-2} m \Gamma\left(\frac{l}{2}\right) \Gamma\left(\frac{1}{2}(l+m+1)\right),
\end{aligned}
$$

where $\Gamma$ are the usual gamma functions (Abramowitz \& Stegun 1972).

\section{D.2. Application to $C_{l, m}$ coefficients}

It is possible to provide a fully analytical expression of $C_{l, m}$ by using Eqs. (D.8), (D.9), (D.16), (D.17). However, it is very cumbersome, and the only interesting tractable expression is obtained in the case when $l=m$, where 


$$
\begin{aligned}
C_{l, l}= & \frac{1}{l(l+1)}\left[A_{l, l}^{2}\left[\left(\alpha_{l, l+1}\right)^{2}+\left(\beta_{l, l+1}\right)^{2}+1\right]\right. \\
& \left.+B_{l, l}^{2}\left[\left(\alpha_{l, l-1}\right)^{2}+\left(\beta_{l, l-1}\right)^{2}+1\right]\right] \\
= & \frac{3+l}{3+5 l+2 l^{2}} .
\end{aligned}
$$

The values of $C_{l, m}$ for the dipolar $(l=1)$ and the quadrupolar $(l=2)$ modes are

$C_{1,0}=\frac{1}{5}, \quad C_{1,1}=C_{1,-1}=\frac{2}{5}$,

$C_{2,0}=\frac{9}{21}, \quad C_{2,1}=C_{2,-1}=\frac{8}{21}, \quad C_{2,2}=C_{2,-2}=\frac{5}{21}$.

\section{D.3. Application to $D_{l, m}$ coefficients}

We have to compute

$D_{l, m}=\int_{\Omega} \sin ^{2} \theta\left|Y_{l}^{m}\right|^{2} \mathrm{~d} \Omega$

(D.21)
Using Eq. (D.4) and the identity

$$
\sin ^{2} \theta=\frac{2}{3}\left(1-\frac{Y_{2}^{0}}{\mathcal{N}_{2}^{0}}\right)
$$

where $\mathcal{N}_{2}^{0}=1 / 2 \sqrt{5 / \pi}$ is obtained with Eq. (D.2), we obtain

$$
D_{l, m}=\frac{2}{3}\left[1-\frac{(-1)^{m}}{\mathcal{N}_{2}^{0}} I_{2, l, l}^{0, m,-m}\right],
$$

where $\mathcal{I}$ has been defined in Eq. (D.13). $(l=2)$ modes are

$$
D_{1,0}=\frac{2}{5}, \quad D_{1,1}=D_{1,-1}=\frac{4}{5},
$$

$D_{2,0}=\frac{10}{21}, \quad D_{2,1}=D_{2,-1}=\frac{4}{7}, \quad D_{2,2}=D_{2,-2}=\frac{6}{7}$ 